\title{
Measure for the Non-Markovianity of Quantum Processes
}

\author{
Elsi-Mari Laine ${ }^{1 *}$, Jyrki Piilo ${ }^{1 \dagger}$, and Heinz-Peter Breuer ${ }^{2 \ddagger}$ \\ ${ }^{1}$ Turku Center for Quantum Physics, Department of Physics and Astronomy, \\ University of Turku, FI-20014, Turun Yliopisto, Finland \\ ${ }^{2}$ Physikalisches Institut, Universität Freiburg, Hermann-Herder-Strasse 3, D-79104 Freiburg, Germany
}

(Dated: October 22, 2018)

\begin{abstract}
Recently, a measure for the non-Markovian behavior of quantum processes in open systems has been developed which is based on the quantification of the flow of information between the open system and its environment [Phys. Rev. Lett. 103, 210401 (2009)]. The information flow is connected to the rate of change of the trace distance between quantum states which can be interpreted in terms of the distinguishability of these states. Here, we elaborate the mathematical details of this theory, present applications to specific physical models, and discuss further theoretical and experimental implications, as well as relations to alternative approaches proposed recently.
\end{abstract}

PACS numbers: 03.65.Yz, 03.65.Ta, 42.50.Lc

\section{INTRODUCTION}

A Markov process in the evolution of an open quantum system typically gives rise to a quantum dynamical semigroup for which the most general representation can be written in the Lindblad form [1, 2. There exist however complex systems for which this relatively simple description of the open system dynamics in terms of a Markovian master equation fails to give a comprehensive picture of the dynamics $[3$. Thus in many realistic physical systems the Markovian approximation of the dynamics gives an overly simplified picture of the open system evolution and a more rigorous treatment of the dynamics is required.

To give insights into the nature of non-Markovian effects many analytical methods and numerical simulation techniques have been developed in recent years (see, for example, Refs. [4-17]). Non-Markovianity manifests itself in the different approaches in a variety of ways and there exists no general recipe for comparing the degree of non-Markovianity in different physical models. In order to give a general quantity determining the degree of nonMarkovian behavior in the open system dynamics, one has to rigorously define what makes a dynamical map non-Markovian.

Here, we discuss a recently proposed measure for the degree of non-Markovian behavior which is based on the trace distance between quantum states [18. The trace distance describes the probability of successfully distinguishing two quantum states and the change in the trace distance of two open system states can be interpreted as a flow of information between the system and the environment. When the trace distance decreases information flows from the system into the environment, while an increase of the trace distance signifies a backflow of information from the environment to the system. Markovian

\footnotetext{
*Electronic address: emelai@utu.fi

${ }^{\dagger}$ Electronic address: jyrki.piilo@utu.fi

${ }^{\ddagger}$ Electronic address: breuer@physik.uni-freiburg.de
}

processes tend to continuously decrease the distinguishability between any two states of the open system, i.e., information flows continuously from the system to the environment. The condition which defines a non-Markovian dynamical map is that the map allows an information flow from the environment to the system and, therefore, allows the system to gain information about its former state. This condition for a non-Markovian map leads to a rigorous and general definition of a measure for the degree of non-Markovianity in open quantum systems.

In Sec. III we construct the measure for nonMarkovianity and discuss its properties for some general classes of quantum processes in open systems. It is shown that the non-divisibility of the dynamical map is necessary for the process to be non-Markovian. Hence, the measure vanishes for quantum dynamical semigroups and for time-dependent Markov processes. We also demonstrate that the appearance of negative rates in the quantum master equation is a necessary condition for nonMarkovianity. In Sec. III we illustrate the determination of the measure for a two-level system and for a $\Lambda$-type atom in a cavity. Section IV] contains a detailed discussion of several alternative ways for defining a measure for non-Markovianity. Moreover, we present possible experimental strategies for the detection of non-Markovian effects. The conclusions are drawn in Sec. $\mathrm{V}$.

\section{THE MEASURE FOR NON-MARKOVIANITY}

\section{A. Construction of the measure}

To construct the measure for non-Markovianity we need a measure for the distance between any pair of quantum states represented by density matrices $\rho_{1}$ and $\rho_{2}$. Such a measure is given by the trace distance, which is defined as

$$
D\left(\rho_{1}, \rho_{2}\right)=\frac{1}{2} \operatorname{Tr}\left|\rho_{1}-\rho_{2}\right|,
$$


where the modulus of an operator $A$ is defined by $|A|=$ $\sqrt{A^{\dagger} A}$. The trace distance $D$ yields a natural metric on the state space and satisfies $0 \leq D \leq 1$. It has many nice properties that make it a useful measure for the distance between quantum states [19. First, the trace distance is preserved under unitary transformations $U$,

$$
D\left(U \rho_{1} U^{\dagger}, U \rho_{2} U^{\dagger}\right)=D\left(\rho_{1}, \rho_{2}\right) .
$$

Second, all completely positive and trace preserving (CPT) maps $\Phi$ (trace preserving quantum operations) are contractions for this metric,

$$
D\left(\Phi \rho_{1}, \Phi \rho_{2}\right) \leq D\left(\rho_{1}, \rho_{2}\right)
$$

Third, the trace distance has a physical interpretation as a measure of state distinguishability. Suppose Alice prepares a quantum system in the state $\rho_{1}$ with probability $1 / 2$, and in the state $\rho_{2}$ with probability $1 / 2$. She gives the system to Bob, who performs a measurement to distinguish the two states. The maximal probability that Bob can identify the state given to him is [20]

$$
p_{\max }=\frac{1}{2}\left[1+D\left(\rho_{1}, \rho_{2}\right)\right] .
$$

Hence, the trace distance represents the maximal bias in favor of the correct state identification which Bob can achieve through an optimal strategy. For example, if $\rho_{1}$ and $\rho_{2}$ have orthogonal supports the trace distance becomes $D\left(\rho_{1}, \rho_{2}\right)=1$ and thus $p_{\max }=1$, which means that Bob is able to distinguish the states with certainty.

The change in the distinguishability of states of an open system can be interpreted as a flow of information between the system and the environment. We consider here quantum processes given by a dynamical CPT map $\Phi(t, 0)$ which transforms the initial states $\rho(0)$ at time zero to the states $\rho(t)$ at time $t \geq 0$,

$$
\rho(0) \mapsto \rho(t)=\Phi(t, 0) \rho(0) .
$$

When such a quantum process reduces the distinguishability of states, information is flowing from the system to the environment. Likewise, the increase of the distinguishability signifies that information flows from the environment to the system. The invariance under unitary transformations (2) indicates that information is preserved under the dynamics of closed systems. The contraction property of Eq. (3) guarantees that the maximal amount of information the system can recover from the environment is the amount of information earlier flowed out the system.

The basic idea underlying our construction for the measure of non-Markovianity in a quantum process is that for Markovian processes information flows continuously from the system to the environment. In order to give rise to non-Markovian effects there must be, for some interval of time, an information flow from the environment back to the system. The information flowing from the environment back to the system allows the earlier states of the system to have an effect on the later dynamics of the system, i.e., it allows the emergence of memory effects.

We define the rate of change of the trace distance of a pair of states by means of

$$
\sigma\left(t, \rho_{1,2}(0)\right)=\frac{d}{d t} D\left(\rho_{1}(t), \rho_{2}(t)\right)
$$

where $\rho_{1,2}(t)=\Phi(t, 0) \rho_{1,2}(0)$. For a non-Markovian process described by a dynamical map $\Phi(t, 0)$, information must flow from the environment to the system for some interval of time and thus we must have $\sigma>0$ for this time interval. A measure of non-Markovianity should measure the total increase of distinguishability over the whole time evolution, i.e., the total amount of information flowing from the environment back to the system. This suggests defining the measure $\mathcal{N}(\Phi)$ for the nonMarkovianity of the quantum process $\Phi(t, 0)$ through

$$
\mathcal{N}(\Phi)=\max _{\rho_{1,2}(0)} \int_{\sigma>0} d t \sigma\left(t, \rho_{1,2}(0)\right) .
$$

The time integration is extended over all time intervals $\left(a_{i}, b_{i}\right)$ in which $\sigma$ is positive and the maximum is taken over all pairs of initial states. Due to Eq. (6) the measure can be written as

$$
\mathcal{N}(\Phi)=\max _{\rho_{1,2}(0)} \sum_{i}\left[D\left(\rho_{1}\left(b_{i}\right), \rho_{2}\left(b_{i}\right)\right)-D\left(\rho_{1}\left(a_{i}\right), \rho_{2}\left(a_{i}\right)\right)\right] .
$$

To calculate this quantity one first determines for any pair of initial states the total growth of the trace distance over each time interval $\left(a_{i}, b_{i}\right)$ and sums up the contribution of all intervals. $\mathcal{N}(\Phi)$ is then obtained by determining the maximum over all pairs of initial states. While it may be difficult to derive an analytical expression for the measure defined in Eq. (8), the numerical evaluation of the measure is relatively easy provided the dynamical map is known explicitly. We will discuss in Sec. III the determination of $\mathcal{N}(\Phi)$ for some specific examples.

\section{B. Classification of quantum processes}

Having defined our measure for non-Markovianity we discuss in this section the properties of this measure for some general classes of quantum processes. Specific physical systems will be investigated in Sec. III.

\section{Divisible maps}

The dynamical map $\Phi(t, 0)$ is defined to be divisible if for all $t, \tau \geq 0$ the CPT map $\Phi(t+\tau, 0)$ can be written as composition of the two CPT maps $\Phi(t+\tau, t)$ and $\Phi(t, 0)$,

$$
\Phi(t+\tau, 0)=\Phi(t+\tau, t) \Phi(t, 0) .
$$

We note that this definition differs slightly from the usual definition of divisibility according to which a CPT map 
$\Lambda$ (quantum channel) is said to be divisible if there exist CPT maps $\Lambda_{1}$ and $\Lambda_{2}$ such that $\Lambda=\Lambda_{1} \Lambda_{2}$, where it is assumed that neither $\Lambda_{1}$ nor $\Lambda_{2}$ is a unitary transformation [21. In Eq. (9) the left-hand side as well as the second factor on the right-hand side are fixed by the given dynamical map. Hence, Eq. (9) requires the existence of a certain linear transformation $\Phi(t+\tau, t)$ which maps the states at time $t$ to the states at time $t+\tau$ and represents a CPT map (that may be a unitary transformation) for all $t$ and all $\tau$. There are many quantum processes which are not divisible. For instance, if $\Phi(t, 0)$ is not invertible, a linear map $\Phi(t+\tau, t)$ which fulfills Eq. (9) may not exist. Moreover, even if a linear map $\Phi(t+\tau, t)$ satisfying Eq. (9) does exist, this map needs not be completely positive, and not even positive.

We claim that all divisible dynamical maps are Markovian. To prove this statement suppose that $\Phi(t, 0)$ is divisible. For any pair of initial states $\rho_{1,2}(0)$ we then have

$$
\rho_{1,2}(t+\tau)=\Phi(t+\tau, t) \rho_{1,2}(t) .
$$

Since $\Phi(t+\tau, t)$ is a CPT map we can apply the contraction property (3) to obtain:

$$
D\left(\rho_{1}(t+\tau), \rho_{2}(t+\tau)\right) \leq D\left(\rho_{1}(t), \rho_{2}(t)\right) .
$$

This shows that for all divisible dynamical maps the trace distance decreases monotonically, i.e., $\sigma\left(t, \rho_{1,2}(0)\right) \leq 0$ and, therefore, $\mathcal{N}(\Phi)=0$. Thus, we conclude that all divisible processes are Markovian and that non-Markovian processes must necessarily be described by a nondivisible dynamical map.

\section{Quantum dynamical semigroups}

The prototype of a Markovian dynamics is provided by a Markovian master equation for the density matrix,

$$
\frac{d}{d t} \rho(t)=\mathcal{L} \rho(t)
$$

with a generator in Lindblad form [1, 2]

$$
\mathcal{L} \rho=-i[H, \rho]+\sum_{i} \gamma_{i}\left[A_{i} \rho A_{i}^{\dagger}-\frac{1}{2}\left\{A_{i}^{\dagger} A_{i}, \rho\right\}\right]
$$

involving a time-independent Hamiltonian $H$ as well as time-independent Lindblad operators $A_{i}$ and positive decay rates $\gamma_{i} \geq 0$. Such a master equation leads to a dynamical semigroup of CPT maps, $\Phi(t, 0)=\exp (\mathcal{L} t)$. With $\Phi(t+\tau, t)=\exp (\mathcal{L} \tau)$ the divisibility condition is trivially satisfied. Hence, we have $\mathcal{N}(\Phi)=0$ for all dynamical semigroups, i.e., for all processes described by a master equation in the Lindblad form.

\section{Time-dependent Markov processes}

The divisibility property holds for a much larger class of quantum processes than those described by a master equation of the form 12. Suppose we have a time-local master equation of the form

$$
\frac{d}{d t} \rho(t)=\mathcal{K}(t) \rho(t)
$$

with a time-dependent generator $\mathcal{K}(t)$. It can be shown that in order to preserve the Hermiticity and trace of the density matrix this generator must be of the form [1, 8]

$$
\begin{aligned}
\mathcal{K}(t) \rho & =-i[H(t), \rho] \\
& +\sum_{i} \gamma_{i}(t)\left[A_{i}(t) \rho A_{i}^{\dagger}(t)-\frac{1}{2}\left\{A_{i}^{\dagger}(t) A_{i}(t), \rho\right\}\right]
\end{aligned}
$$

By contrast to the assumptions in Eq. 13 the Hamiltonian $H(t)$, the Lindblad operators $A_{i}(t)$ and the decay rates $\gamma_{i}(t)$ may now depend on time. If the decay rates are positive functions, $\gamma_{i}(t) \geq 0$, the generator 15 is in Lindblad form (13) for each fixed $t \geq 0$. Such a process with $\gamma_{i}(t) \geq 0$ may be called time-dependent Markovian although the corresponding dynamical map

$$
\Phi(t, 0)=\mathrm{T} \exp \left[\int_{0}^{t} d t^{\prime} \mathcal{K}\left(t^{\prime}\right)\right]
$$

does not yield a dynamical semigroup ( $\mathrm{T}$ denotes the chronological time-ordering operator). However, one can easily see that the divisibility condition (9) still holds because the map

$$
\Phi(t+\tau, t)=\mathrm{T} \exp \left[\int_{t}^{t+\tau} d t^{\prime} \mathcal{K}\left(t^{\prime}\right)\right]
$$

is CPT for $\gamma_{i}(t) \geq 0$. Thus we can conclude that for all time-dependent Markovian processes we again have $\mathcal{N}(\Phi)=0$.

We have just seen that a quantum process given by the time-local master equation (14) with positive rates leads to a divisible dynamical map. Under certain conditions the converse of this statement is also true. More precisely, if the dynamical map $\Phi(t, 0)$ is divisible with a unique map $\Phi(t+\tau, t)$ depending smoothly on $\tau$, then the corresponding density matrix $\rho(t)$ obeys a master equation of the form (14) with positive rates in the generator 15. In fact, using $\rho(t+\tau)=\Phi(t+\tau, t) \rho(t)$ we find

$$
\frac{d}{d t} \rho(t)=\left.\frac{d}{d \tau}\right|_{\tau=0} \Phi(t+\tau, t) \rho(t)
$$

and, hence, we obtain the master equation (14), where the generator is given by

$$
\mathcal{K}(t)=\left.\frac{d}{d \tau}\right|_{\tau=0} \Phi(t+\tau, t)
$$

Since $\Phi(t+\tau, t)$ is CPT and satisfies $\Phi(t, t)=I$, this generator must be in Lindblad form for each fixed $t$, i.e., it must have the form 15 with $\gamma_{i}(t) \geq 0$. 


\section{Non-Markovian processes}

The measure for quantum non-Markovianity does not depend on any specific mathematical representation of the dynamics. There are many different such representations, e.g., through generalized master equations involving a certain memory kernel. However, quantum master equations with the time-local structure given by Eqs. (14) and (15) are also very useful for the description of nonMarkovian processes. It follows from the preceding results that in order for such a master equation to yield a nonzero measure, $\mathcal{N}(\Phi)>0$, at least one of the rates $\gamma_{i}(t)$ must take on negative values for some interval of time. We emphasize that temporarily negative rates in the master equation do in general not lead to a violation of the complete positivity of the dynamical map. Many examples for time-local master equations with negative rates are known in the literature. Further examples will be discussed in the next section.

\section{EXAMPLES}

\section{A. Two-level system}

We study the dynamics of a two-level atom with excited state $|+\rangle$ and ground state $|-\rangle$ which is coupled to a reservoir of field modes initially in the vacuum state. In Ref. 18 we have described the detuned JaynesCummings model, while here we treat the resonant case. We will show that the pair of states maximizing the measure for non-Markovinity is different in the two cases. This demonstrates that the change in both the populations and the coherences plays a crucial role in the flow of information between the system and the environment.

The two-level atom model can easily be solved exactly [3] and leads to a dynamical map $\Phi(t, 0)$ which can be represented in terms of the elements $\rho_{ \pm \pm}(t)=\langle \pm|\rho(t)| \pm\rangle$ of the density matrix $\rho(t)$ as follows,

$$
\begin{aligned}
& \rho_{++}(t)=|G(t)|^{2} \rho_{++}(0) \\
& \rho_{--}(t)=\rho_{--}(0)+\left(1-|G(t)|^{2}\right) \rho_{++}(0), \\
& \rho_{+-}(t)=G(t) \rho_{+-}(0) \\
& \rho_{-+}(t)=G^{*}(t) \rho_{-+}(0)
\end{aligned}
$$

Here, the function $G(t)$ is defined as the solution of the integrodifferential equation

$$
\frac{d}{d t} G(t)=-\int_{0}^{t} d t_{1} f\left(t-t_{1}\right) G\left(t_{1}\right)
$$

corresponding to the initial condition $G(0)=1$, where $f\left(t-t_{1}\right)$ denotes the two-point reservoir correlation function (Fourier transform of the spectral density). The map (20) is completely positive if and only if $|G(t)| \leq 1$. One can easily check that $\Phi(t, 0)$ can be decomposed as in
Eq. (9), where the map $\Phi(t+\tau, t)$ is given by

$$
\begin{aligned}
\rho_{++}(t+\tau) & =\left|\frac{G(t+\tau)}{G(t)}\right|^{2} \rho_{++}(t), \\
\rho_{--}(t+\tau) & =\rho_{--}(t)+\left(1-\left|\frac{G(t+\tau)}{G(t)}\right|^{2}\right) \rho_{++}(t), \\
\rho_{+-}(t+\tau) & =\frac{G(t+\tau)}{G(t)} \rho_{+-}(t), \\
\rho_{-+}(t+\tau) & =\frac{G^{*}(t+\tau)}{G^{*}(t)} \rho_{-+}(t) .
\end{aligned}
$$

It follows from these equations that a necessary and sufficient condition for the complete positivity of $\Phi(t+\tau, t)$ is given by $|G(t+\tau)| \leq|G(t)|$. Thus we see that the dynamical map of the model is divisible if and only if $|G(t)|$ is a monotonically decreasing function of time. Note that this statement holds true also for the case that $G(t)$ vanishes at some finite time.

With the help of the above results one can easily derive an analytical formula for the time derivative of the trace distance,

$$
\sigma\left(t, \rho_{1,2}(0)\right)=\frac{2|G(t)|^{2} a^{2}+|b|^{2}}{\sqrt{|G(t)|^{2} a^{2}+|b|^{2}}} \frac{d}{d t}|G(t)|,
$$

where $a=\rho_{1}^{++}(0)-\rho_{2}^{++}(0)$ denotes the difference of the populations and $b=\rho_{1}^{+-}(0)-\rho_{2}^{+-}(0)$ the difference of the coherences of the initial states. This relation shows that the trace distance increases at some point if and only if $|G(t)|$ increases at this point. We conclude that the measure for non-Markovianity is positive, $\mathcal{N}(\Phi)>0$, if and only if the dynamical map is nondivisible.

A positive measure for non-Markovianity is not only linked to a breakdown of the divisibility of the dynamical map, but also to the emergence of a negative rate in the corresponding master equation (14). In fact, as long as $G(t) \neq 0$ one can write an exact master equation of this form with the generator

$$
\begin{aligned}
\mathcal{K}(t) \rho= & -\frac{i}{2} S(t)\left[\sigma_{+} \sigma_{-}, \rho\right] \\
& +\gamma(t)\left[\sigma_{-} \rho \sigma_{+}-\frac{1}{2}\left\{\sigma_{+} \sigma_{-}, \rho\right\}\right],
\end{aligned}
$$

where we use the definitions

$$
\gamma(t)=-2 \Re\left(\frac{\dot{G}(t)}{G(t)}\right), \quad S(t)=-2 \Im\left(\frac{\dot{G}(t)}{G(t)}\right) .
$$

Writing the rate $\gamma(t)$ as

$$
\gamma(t)=-\frac{2}{|G(t)|} \frac{d}{d t}|G(t)|
$$

we see that an increase of $|G(t)|$ and, hence, a breakdown of the divisibility leads to a negative rate in the generator of the master equation. Thus we find that for the present 


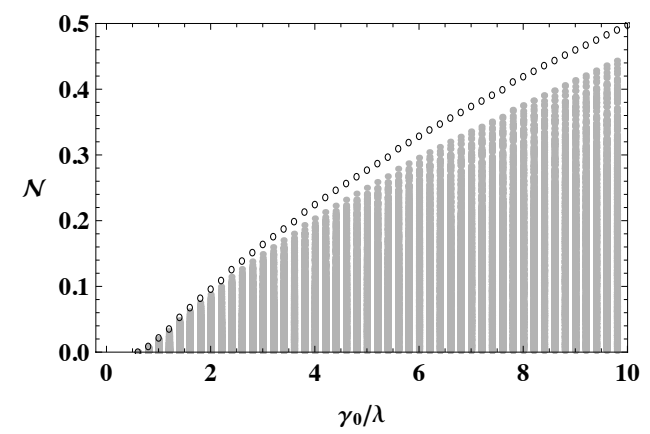

FIG. 1: The non-Markovianity $\mathcal{N}(\Phi)$ for the damped JaynesCummings model as a function of the coupling strength $\gamma_{0}$. Gray dots: 1000 randomly drawn pairs of pure and mixed initial states. Black circles: The initial pair given by Eq. 29. which leads to the maximum in Eq. (7).

model a nonzero measure for non-Markovianity is equivalent to the non-divisibility of the dynamical map and to the occurrence of a negative rate in the master equation.

As an example we consider the case of a Lorentzian reservoir spectral density which is on resonance with the atomic transition frequency and leads to an exponential two-point correlation function

$$
f(\tau)=\frac{1}{2} \gamma_{0} \lambda e^{-\lambda|\tau|},
$$

where $\gamma_{0}$ describes the coupling strength and $\lambda$ the spectral width (damped Jaynes-Cummings model). Solving Eq. (21) with this correlation function we find

$$
G(t)=e^{-\lambda t / 2}\left[\cosh \left(\frac{d t}{2}\right)+\frac{\lambda}{d} \sinh \left(\frac{d t}{2}\right)\right],
$$

where $d=\sqrt{\lambda^{2}-2 \gamma_{0} \lambda}$. We see that for small couplings, $\gamma_{0}<\lambda / 2$, the function $|G(t)|$ decreases monotonically. The dynamical map is thus divisible in the weak coupling regime, the rate $\gamma(t)$ is positive, and the measure for nonMarkovianity vanishes. However, in the strong coupling regime, $\gamma_{0}>\lambda / 2$, the function $|G(t)|$ starts to oscillate, showing a non-monotonic behavior. Consequently, the dynamical map is then no longer divisible and $\mathcal{N}(\Phi)>0$. We note that in the strong coupling regime the rate $\gamma(t)$ diverges at the zeros of $G(t)$. However, the master equation can still be used to describe the evolution between successive zeros and, therefore, the connection between a positive measure and negative rates in the master equation remains valid.

There is thus a threshold $\gamma_{0}=\lambda / 2$ for the systemreservoir coupling below which $\mathcal{N}(\Phi)=0$. We find that the measure increases monotonically with increasing coupling for $\gamma_{0}>\lambda / 2$. This is illustrated in Fig. 1. The maximization over the pair of initial states $\rho_{1,2}(0)$ in expression (7) has been performed here by a Monte Carlo sampling of pairs of initial states. Our simulations provide strong evidence that the maximum is attained for the initial states

$$
\rho_{1}(0)=|-\rangle\left\langle-\left|, \quad \rho_{2}(0)=\frac{1}{2}(|+\rangle+|-\rangle)(\langle+|+\langle-|) .\right.\right.
$$

In Ref. [18, we calculated the measure for the detuned Jaynes-Cummings model in the weak coupling limit. In this example the maximum of the measure was obtained for the initial states $\rho_{1}(0)=|-\rangle\langle-|$ and $\rho_{2}(0)=|+\rangle\langle+|$, i.e. for the invariant ground state and the excited state. The difference in the maximization for the resonant and the off-resonant case arises from the fact that the rate at which the populations and the coherences initially decay is much larger for the resonant case. Consequently, the growth of the trace distance occurs after the excited state population and the coherences have reached the value zero. After this point, the increase of the coherences yields the dominant contribution to the increase of the trace distance. Therefore, the maximal growth of the trace distance for the resonant case is reached for the invariant state and the state with maximal initial coherence.

\section{B. $\Lambda$-model}

The $\Lambda$-model describes a three-level atom with excited state $|a\rangle$ and two ground states $|b\rangle$ and $|c\rangle$ interacting off-resonantly with a cavity field. This example allows us to demonstrate how the measure for non-Markovianity operates in a multi-channel case and how there can exist simultaneously positive and negative decay rates for different channels. The spectral density we use is

$$
J(\omega)=\frac{\gamma_{0}}{2 \pi} \frac{\lambda^{2}}{\left(\omega_{\text {cav }}-\omega\right)^{2}+\lambda^{2}},
$$

where $\omega_{\text {cav }}$ is the resonance frequency of the cavity. Further details and the master equation describing the dynamics of the $\Lambda$-type atom are presented in the Appendix. The generator of the master equation is of the form of Eq. 15) with two Lindblad operators $|b\rangle\langle a|$ and $|c\rangle\langle a|$, and two time-dependent decay rates $\gamma_{1}(t)$ and $\gamma_{2}(t)$.

The detunings of the transition frequencies of the $\Lambda$ atom from the cavity resonance frequency are denoted by $\Delta_{i}=\omega_{i}-\omega_{\text {cav }}$. When the detuning parameters $\Delta_{1}$ and $\Delta_{2}$ are both sufficiently large, the decay rates $\gamma_{1}(t)$ and $\gamma_{2}(t)$ get temporarily negative values and this gives rise to an information flow from the environment to the system. On one hand, the two decay rates $\gamma_{1}(t)$ and $\gamma_{2}(t)$ have simultaneous negative regions when $\Delta_{1}=\Delta_{2}$. On the other hand, when $\Delta_{1} \neq \Delta_{2}$, the decay rates can have opposite signs. In this case, the co-operative action of the other channel reduces the amount of information flowing from the environment to the system. The maximum of the measure over the initial states is reached when the states are chosen to be $|a\rangle\langle a|$ and $|b\rangle\langle b|$, or $|a\rangle\langle a|$ and $|c\rangle\langle c|$, depending on which of the channels has more information flow from the environment to the system. When 
$\Delta_{1}$ and $\Delta_{2}$ are such that the channel corresponding to the decay rate $\gamma_{i}(t)(i=1$ or 2$)$ causes more information flow from the environment to the system we get the expression

$$
\sigma(t)=-\gamma_{i}(t) \rho_{a a}(t)
$$

The function $\rho_{a a}(t)$ is specified in the Appendix. Eq. (31) shows that the $\Lambda$-system is non-Markovian if one of the decay rates $\gamma_{1}(t)$ or $\gamma_{2}(t)$ takes on negative values. The maximization over the the pair of initial states is demonstrated in Fig. 2, where the measure was again calculated numerically from a large sample of initial states.

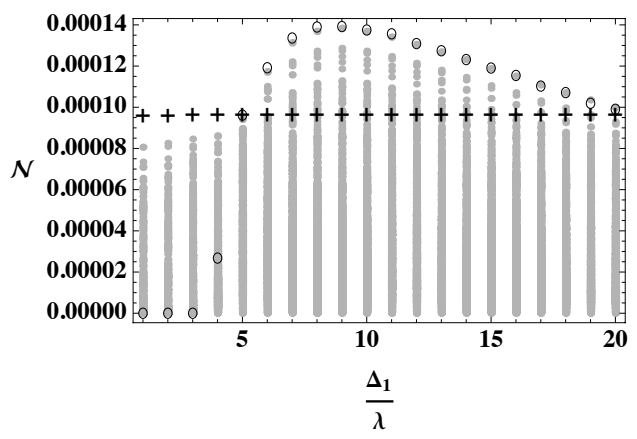

FIG. 2: The non-Markovianity $\mathcal{N}(\Phi)$ for the $\Lambda$-model as a function of the detuning $\Delta_{1}$ for $\Delta_{2} / \lambda=5$ and $\gamma_{0} / \lambda=0.01$. Gray dots: 1000 randomly drawn pairs of initial states. Circles: The initial pair $\rho_{1}(0)=|a\rangle\langle a|$ and $\rho_{2}(0)=|b\rangle\langle b|$. Pluses: The initial pair $\rho_{1}(0)=|a\rangle\langle a|$ and $\rho_{2}(0)=|c\rangle\langle c|$. At $\Delta_{1}=\Delta_{2}$ the pair which yields the maximum in Eq. (7) changes from the latter to the former pair of initial states.

\section{DISCUSSION}

\section{A. Alternative distance measures}

We have based our definition of the measure of nonMarkovianity on the trace distance (1). An alternative measure is obtained if one replaces the trace distance by the relative entropy

$$
S\left(\rho_{1} \| \rho_{2}\right)=\operatorname{Tr}\left[\rho_{1}\left(\log \rho_{1}-\log \rho_{2}\right)\right] .
$$

Using this quantity as a measure for the distance between quantum states one is led to a similar interpretation as before because the relative entropy also decreases under CPT maps 22. There are however some technical problems and limitations in the usefulness of the relative entropy which arise from the fact that for many pairs $\rho_{1}$ and $\rho_{2}$ the relative entropy becomes infinite [23] and thus leads to singularities in the definition of the measure. This situation can occur even in the simple case of a two-state system, demonstrating the problems of the relative entropy concept in defining a general measure for non-Markovianity. No such problems occur for the trace distance which is well-defined and finite for all physical states represented by positive trace class operators.

Another common measure for the distance between two states is the Hilbert-Schmidt distance

$$
D_{\mathrm{HS}}\left(\rho_{1}, \rho_{2}\right)=\sqrt{\operatorname{Tr}\left[\left(\rho_{1}-\rho_{2}\right)^{2}\right]} .
$$

For two-dimensional Hilbert spaces the Hilbert-Schmidt distance and the trace distance coincide and correspond to the Euclidean distance between the Bloch vectors representing the states (up to numerical factors). However, the Hilbert-Schmidt distance is not suitable for a definition of non-Markovianity since CPT maps are in general not contractions for this metric [24. Thus, the HilbertSchmidt distance does not provide a natural way to define the information flow between system and environment.

\section{B. Experimental issues}

The exact determination of the measure generally requires solving the complete reduced dynamics which can be a difficult task for more complex systems. However, any observed growth of the trace distance is a clear signature for non-Markovian behavior and leads to a lower bound for $\mathcal{N}(\Phi)$. The measure for non-Markovianity introduced here could therefore be useful also for the experimental detection of non-Markovianity.

In an experiment one has to perform a state tomography on different ensembles at different times in order to decide whether or not the trace distance has increased. Such an experiment also allows the validation of theoretical models or approximation schemes. Consider a theoretical model predicting $\sigma\left(t, \rho_{1,2}(0)\right)>0$ for some interval $t \in\left(t_{1}, t_{2}\right)$ and for some pair of initial states $\rho_{1,2}(0)$. In the experiment one should then detect the increase of the trace distance between the states $\rho_{1}(t)$ and $\rho_{2}(t)$ in this time interval. This type of experiment could be based, e. g., on the recent proposal to use a trapped ion to study quantum Brownian motion in the non-Markovian regime [25. The explicit experimental implementation of this system can be done, e. g., by using reservoir engineering techniques [26] or by using the trapped ion as a quantum simulator for non-Markovian dynamics [27]. One of the possibilities here to detect non-Markovianity is to prepare the ion in various Fock states, and to study the trace distance dynamics as described above.

A great advantage of the present approach is that it also allows to plan experiments for testing nonMarkovianity without knowing the properties of the environment or the system-environment interaction. The interactions and environmental properties can be quite difficult to model in an experimental setup. By performing a state tomography for two states of the open system under study at many different times, one can determine whether there has been any increase in the trace distance and, hence, non-Markovian behavior in the dynamics. From this information one can conclude whether or not non-Markovian effects are crucial in the dynamics and 
in this way also gain some knowledge on the nature of the environment and the interactions. An example under active investigation, where nevertheless a complete characterization of the environment is still missing and where non-Markovianity could play a role, is given by the energy transfer in photosynthetic systems [28].

\section{Other approaches to non-Markovianity}

Recently, other interesting approaches to the characterization and quantification of non-Markovianity have been proposed. The measure suggested in Ref. [5] quantifies non-Markovianity in terms of the minimal amount of noise required to make a given quantum channel Markovian. The most important difference to our approach is that this measure is based on the properties of the dynamical map at a given time, i.e., on the properties of the quantum channel represented by a snapshot of the time evolution. Hence, this approach assesses to what extend the dynamical map at each fixed time $t_{0}$ deviates from an element of a Markovian process. The fundamental difference between the notion of non-Markovianity used in Ref. [5] and ours can be seen from the following simple example. We consider the dynamical map $\Phi(t, 0)$ of a two state system undergoing a pure de- and re-phasing dynamics which is given by (using the notation of Sec. III A)

$$
\begin{aligned}
& \rho_{++}(t)=\rho_{++}(0), \quad \rho_{--}(t)=\rho_{--}(0) \\
& \rho_{+-}(t)=g(t) \rho_{+-}(0), \quad \rho_{-+}(t)=g(t) \rho_{-+}(0)
\end{aligned}
$$

where the function $g(t)=\frac{1}{2}\left[1+\cos ^{2} \omega t\right]$ describes a periodic oscillation of the coherences. The trace distance for this model is given by

$$
D\left(\rho_{1}(t), \rho_{2}(t)\right)=\sqrt{a^{2}+g^{2}(t)|b|^{2}}
$$

where $a=\rho_{1}^{++}(0)-\rho_{2}^{++}(0)$ and $b=\rho_{1}^{+-}(0)-\rho_{2}^{+-}(0)$. For $b \neq 0$ the trace distance thus oscillates periodically and, hence, $\mathcal{N}(\Phi)=+\infty$ according to the definition (7) of our measure. On the other hand, the non-Markovianity in the sense of Ref. [5] is zero because for any fixed $t_{0}$ the dynamical map (34) can be written as an element of a Markovian semigroup: $\Phi\left(t_{0}, 0\right)=\exp (\mathcal{L})$ with the Lindblad generator $\mathcal{L} \rho=\frac{\Gamma}{2}\left(\sigma_{3} \rho \sigma_{3}-\rho\right)$, where $\Gamma=-\ln g\left(t_{0}\right)$.

A further interesting measure proposed recently [ 6 ] is closely connected to the measure discussed here. In fact, the measure of Ref. 6] quantifies deviations from the divisibility of the dynamical map. As we have seen, the non-divisibility of the dynamical map is a necessary condition for $\mathcal{N}(\Phi)$ to be nonzero. However, we conjecture that our notion of non-Markovianity and the one used in [6] are not strictly equivalent, i.e., that there are nondivisible maps with $\mathcal{N}(\Phi)=0$. Further considerations concerning this point will be published elsewhere.

\section{CONCLUSIONS}

We have constructed a measure $\mathcal{N}(\Phi)$ for the nonMarkovianity of quantum processes in open systems in terms of the information flowing from the environment to the system during the time evolution. The flow of information is characterized by the change of the distinguishability between a pair of quantum states which, in turn, is linked to the change of the trace distance between these states. We have also argued why the trace distance represents the most suitable distance measure for quantum states to be used in this context. Furthermore, since we have developed a genuine quantitative measure, the results presented here also allow to compare the degree of non-Markovianity of different types of physical systems.

It has been demonstrated that a nonzero measure for non-Markovianity requires the dynamical map to be nondivisible, a property which is thus necessary for the presence of memory effects in the open system dynamics. It has also been shown that Markovian semigroups and time-dependent Markov processes are divisible and, hence, lead to $\mathcal{N}(\Phi)=0$. The examples discussed here illustrate how the measure can be calculated for a given open system dynamics and that a nonzero measure for non-Markovianity is linked to the emergence of negative decay rates in the corresponding master equation.

Our measure for non-Markovianity has a clear operational meaning based on the interpretation of the trace distance in terms of the distinguishability of states, and suggests various ways to experimentally decide whether a system under study is non-Markovian. The measurement scheme discussed here has the great advantage that it does not presuppose any knowledge about the structure of the environment or about the system-environment interaction and, therefore, also gives valuable information on the theoretical modelling of the open system dynamics. If, for example, a substantial increase of the trace distance is observed experimentally, a mathematical description of the dynamics through any equation describing a Markovian or time-dependent Markovian process is excluded. This shows that our measure is a useful tool for the characterization of non-Markovianity, both in experiments on open systems and in their theoretical analysis and modelling.

We have argued that the characteristics of the information exchange between the system and its environment determine the degree of non-Markovian behavior in an open system. This exchange of quantum information has been defined here in very general terms through the change of the distinguishability of quantum states, and does not presuppose anything about the specific physical carriers of the information, e.g., energy or particles. Moreover, the measure does not depend on any specific representation of the open system's dynamics. It therefore opens the possibility to compare and assess different mathematical formulations of dynamical processes in their ability to describe memory effects, in order to understand better the mathematical description of non- 
Markovian quantum dynamics.

\section{Acknowledgments}

The authors thank the Academy of Finland (projects 133682, 115982, 115682), Magnus Ehrnrooth Foundation, and the Finnish National Graduate School of Modern Optics and Photonics for financial support.

\section{Appendix}

Here we present some details of the $\Lambda$-model studied in Sec. IIIB. The weak-coupling master equation for this model is given by

$$
\begin{aligned}
\frac{d}{d t} \rho(t)= & -i \lambda_{1}(t)[|a\rangle\langle a|, \rho(t)]-i \lambda_{2}(t)[|a\rangle\langle a|, \rho(t)] \\
& +\gamma_{1}(t)\left[|b\rangle\langle a|\rho(t)| a\rangle\langle b|-\frac{1}{2}\{\rho(t),|a\rangle\langle a|\}\right] \\
& +\gamma_{2}(t)\left[|c\rangle\langle a|\rho(t)| a\rangle\langle c|-\frac{1}{2}\{\rho(t),|a\rangle\langle a|\}\right],
\end{aligned}
$$

where

$$
\begin{aligned}
& \lambda_{i}(t)=\int_{0}^{t} d s \int_{0}^{\infty} d \omega J(\omega) \sin \left[\left(\omega-\omega_{i}\right) s\right], \\
& \gamma_{i}(t)=\int_{0}^{t} d s \int_{0}^{\infty} d \omega J(\omega) \cos \left[\left(\omega-\omega_{i}\right) s\right] .
\end{aligned}
$$

Introducing the definitions

$$
\begin{aligned}
f(t) & =e^{-\left[D_{1}(t)+D_{2}(t)\right] / 2} e^{-i\left[L_{1}(t)+L_{2}(t)\right]}, \\
g_{i}(t) & =\int_{0}^{t} d s \gamma_{i}(s) e^{-\left[D_{1}(s)+D_{2}(s)\right]},
\end{aligned}
$$

where

$$
D_{i}(t)=\int_{0}^{t} d s \gamma_{i}(s), \quad L_{i}(t)=\int_{0}^{t} d s \lambda_{i}(s)
$$

the solution of the master equation can be represented as follows,

$$
\begin{aligned}
\rho_{a a}(t) & =|f(t)|^{2} \rho_{a a}(0), \\
\rho_{b b}(t) & =g_{1}(t) \rho_{a a}(0)+\rho_{b b}(0), \\
\rho_{c c}(t) & =g_{2}(t) \rho_{a a}(0)+\rho_{c c}(0), \\
\rho_{a b}(t) & =f(t) \rho_{a b}(0), \\
\rho_{a c}(t) & =f(t) \rho_{a c}(0), \\
\rho_{b c}(t) & =\rho_{b c}(0) .
\end{aligned}
$$

These equations define the dynamical map $\Phi(t, 0)$ of the $\Lambda$-model. Employing the results of Choi [29] one can check that a necessary and sufficient condition for the complete positivity of this map is given by

$$
g_{1}(t) \geq 0, \quad g_{2}(t) \geq 0
$$

These conditions are satisfied for the parameters used in the simulations of Sec. IIIB.
[1] V. Gorini, A. Kossakowski and E. C. G. Sudarshan, J. Math. Phys. 17, 821 (1976).

[2] G. Lindblad, Comm. Math. Phys. 48, 119 (1976).

[3] H. P. Breuer and F. Petruccione, The Theory of Open Quantum Systems (Oxford University Press, Oxford, 2002).

[4] J. Piilo, S. Maniscalco, K. Härkönen and K.-A. Suominen, Phys. Rev. Lett. 100, 180402 (2008); J. Piilo, K. Härkönen, S. Maniscalco and K.-A. Suominen, Phys. Rev. A 79, 062112 (2009); H. P. Breuer and J. Piilo, Europhys. Lett. 85, 50004 (2009).

[5] M. M. Wolf, J. Eisert, T. S. Cubitt and J. I. Cirac, Phys.Rev. Lett. 101, 150402 (2008).

[6] A. Rivas, S. F. Huelga and M. B. Plenio (2009), eprint arXiv:0911.4270v1.

[7] H. P. Breuer and B. Vacchini, Phys. Rev. Lett. 101, 140402 (2008); Phys. Rev. E 79, 041147 (2009).

[8] H. P. Breuer, Phys. Rev. A 70, 012106 (2004).

[9] D. Chruściński, A. Kossakowski and S. Pascazio, Phys.
Rev. A 81, 032101 (2010).

[10] H. Krovi, O. Oreshkov, M. Ryazanov and D. A. Lidar, Phys. Rev. A 76, 052117 (2007).

[11] A. J. van Wonderen and K. Lendi, Europhys. Lett. 71, 737 (2006).

[12] A. A. Budini, Phys. Rev. A 74, 053815 (2006).

[13] S. M. Barnett and S. Stenholm, Phys. Rev. A 64,033808 (2001).

[14] J. Wilkie and Y. M. Wong, J. Phys. A 42, 015006 (2009).

[15] S. Daffer, K. Wódkiewicz, J. D. Cresser and J. K. McIver, Phys. Rev. A 70, 010304(R) (2004).

[16] A. Kossakowski and R. Rebolledo, Open Syst. Inf. Dyn. 15, 135 (2008); Open Syst. Inf. Dyn. 16, 259 (2009).

[17] A. Shabani and D. A. Lidar, Phys. Rev. Lett. 102, 100402 (2009).

[18] H. P. Breuer, E.-M. Laine, J. Piilo, Phys. Rev. Lett. 103, 210401 (2009).

[19] M. A. Nielsen and I. L. Chuang, Quantum Computation and Quantum Information (Cambridge University Press, 
Cambridge, 2000).

[20] A. Gilchrist, N. K. Langford and M. A. Nielsen, Phys. Rev. A 71, 062310 (2005).

[21] M. M. Wolf and J. I. Cirac, Commun. Math. Phys, 279, 147 (2008).

[22] G. Lindblad, Commun. Math. Phys. 40, 147 (1975).

[23] K. Lendi, J. Stat. Phys. 50, 1103 (1988).

[24] X. Wang and S. G. Schirmer, Phys. Rev. A 79, 052326 (2009).

[25] S. Maniscalco, J. Piilo, F. Intravaia, F. Petruccione and A. Messina, Phys. Rev. A 69, 052101 (2004).
[26] C. J. Myatt, B. E. King, Q. A. Turchette, C. A. Sackett, D. Kielpinski, W. M. Itano, C. Monroe, D. J. Wineland, Nature 403, 269 (2000).

[27] J. Piilo and S. Maniscalco, Phys. Rev. A 74, 032303 (2006).

[28] G. S. Engel, T. R. Calhoun, E. L. Read, T.-K. Ahn, T. Mančal, Y.-C. Cheng, R. E. Blankenship, and G. R. Fleming, Nature 446, 782 (2007).

[29] M. D. Choi, Linear Algebra Appl. 10 , 285 (1975). 